\title{
Kommunikation über Evaluationen zu Umweltfragen - Ergebnisse einer Session des AK Umwelt bei der DeGEval-Jahrestagung 2020
}

\author{
Dominik Jessing, ${ }^{1}$ Christelle Nowack, ${ }^{2}$ Reinhard Zweidler ${ }^{3}$
}

Der Arbeitskreis Umwelt hat bei der Jahrestagung der DeGEval 2020 eine Session (D1) durchgeführt, an der 31 Personen teilnahmen. Genau wie der Rest der Tagung fand die Veranstaltung in virtueller Form statt.

$\mathrm{Zu}$ Beginn der Session führten zwei Vorträge über Herausforderungen und Möglichkeiten der Kommunikation über Evaluationen zu Umweltfragen beispielhaft in das Tagesthema ein.

\section{Nutzung von Fachsprache}

Im ersten Vortrag stellte Reinhard Zweidler drei Beispiele vor, bei denen durch die Nutzung von Fachsprache Fehler in der Kommunikation entstanden sind:

1. „Juristisch“ ist nicht gleich Deutsch: In diesem Beispiel geht es um die Verwendung von Alltagsbegriffen in der Gesetzgebung der Schweiz, denen im rechtlichen Zusammenhang eine andere Bedeutung zukommt. Die Evaluationsfrage lautete, ob Aufgaben, die der Bund in der Umweltbildung wahrnimmt, gestrichen werden können. Im Gesetz steht, dass der Bund in diesem Bereich Aufgaben übernehmen „kann“ - was im juristischen Sinne in diesem Fall bedeutet, dass die Aufgaben vom Bund wahrgenommen werden „müssen“ und nicht gestrichen werden können. Durch die fehlende Vertrautheit mit der spezifischen juristischen Fachsprache entstand im vorgelegten Fall ein falsches Evaluationsergebnis, das die Streichung der Umweltbildung empfahl.

2. Fachsprache ist nicht gleich Deutsch: Im zweiten Beispiel ging es um die Frage, ob im Zusammenhang mit einem konkreten Windkraftprojekt, Maßnahmen zum Schutz des Rotmilans getroffen werden müssen. Er steht zwar in der Schweiz nicht auf der roten Liste der gefährdeten Arten, aber auf der Liste der national prioritären Arten. Ohne das nötige Fachwissen ist unklar, welche der Listen für

1 ifeu - Institut für Energie- und Umweltforschung Heidelberg gGmbH

2 DLR Projektträger, Bereich Umwelt und Nachhaltigkeit, Bonn

3 EBP Schweiz AG, Zollikon 
den Schutzstatus des Rotmilans maßgeblich ist. Im konkreten Fall ist das die Liste der prioritären Arten, was letztlich definiert, welche Art von Schutzmaßnahmen getroffen werden müssen.

Die Beispiele 1 und 2 zeigen, dass Evaluierende entweder das nötige Fachwissen selbst haben oder erkennen müssen, wann entsprechende Fachleute/Projektbeteiligte hinzugezogen werden sollten. Gleichzeitig sollen Evaluierende möglichst neutral sein/bleiben, was durch Abstimmungen mit Projektbeteiligten strenggenommen beeinträchtigt wird.

3. Evaluationssprache ist nicht gleich Deutsch: Das letzte Beispiel zeigt eine Ergebnispräsentation einer Evaluation. Evaluierende nutzten dabei Evaluationsfachsprache und wurden von relevanten Akteur(inn)en nicht verstanden. Das Problem verschärfte sich im vorgelegten Beispiel dadurch, dass das mangelnde Verständnis durch die evaluationsfremde Projektleitung nicht benannt wurde („Ich möchte nicht als ungebildet wahrgenommen werden“), mit der Folge, dass die für die Interpretation der Evaluationsergebnisse nötigen Informationen nicht übermittelt werden konnten. Nur wenige Personen arbeiten sich in die Fachsprache ein und bringen dadurch vielleicht überproportional stark ihre Partikularinteressen ein.

Das dritte Beispiel zeigt den Bedarf, Evaluationsfachsprache zu vereinheitlichen und möglichst für die breite Öffentlichkeit zu erklären und damit zugänglich zu machen. Diese Aufgabe hat die DeGEval (zusammen mit der SEVAL) z.B. über das Glossar bereits in Angriff genommen. Parallel zur Entwicklung einer einheitlichen Fachsprache müssen Evaluierende darin geschult und zur Nutzung (selbst-)verpflichtet werden.

\section{Wie kann Evaluation in der Gesellschaft besser wahrgenommen werden? Ein Fallbeispiel aus der Schweiz}

Im zweiten Vortrag analysierte Yves Steiner die Frage, welche Kommunikation hilft, damit Evaluationsergebnisse wahrgenommen werden. Dazu schilderte er die Lage der Eidgenössischen Finanzkontrolle EFK (vergleichbar mit dem deutschen Rechnungshof). Über viele Jahre wurden Evaluationsberichte der EFK in der Öffentlichkeit wenig wahrgenommen. Damit fehlte im schweizerischen politischen System aber oft ein Korrektiv durch die Gesellschaft. Die EFK hat sich intensiv mit der Frage auseinandergesetzt, wie die öffentliche Wirkung von Evaluationsergebnissen verbessert werden kann und sich entschieden, auf einige Dinge besonders zu achten. Die neue Kommunikationsstrategie in der EFK beinhaltet u.a.:

- viel mehr zu publizieren. U.a. werden nun Jahresberichte und das Programm für Publikationen mindestens sechs Mal pro Jahr veröffentlicht. Die neue Kommunikationsstrategie ist auch gekennzeichnet durch eine starke Nutzung des Internets und schnelle Beantwortung von Anfragen. 
- ein Presse-Embargo nach Veröffentlichungen von drei Tagen. Ziel ist es, Journalist(inn)en die nötige Zeit einzuräumen, sich mit der Veröffentlichung zu befassen, bevor Anfragen beantwortet werden.

- sehr kurze Zusammenfassungen von Evaluationsberichten (max. zwei Seiten) und die aktive Vermeidung der „Blabla-Falle“.

- Trainingsprogramme für Auditierende und Evaluierende.

- eine Evaluation der Kommunikationsstrategie durch externe Media-Expert(inn)en.

Die Umsetzung der Kommunikationsstrategie ist mit einigem Aufwand für die Akteurinnen und Akteure verbunden, erscheint aber nötig.

\section{Sechs Thesen rund um Kommunikation bei Naturschutzevaluationen}

Ein anderes Fallbeispiel stellte Christelle Nowack (DLR Projektträger, Programmbüro des Bundesamtes für Naturschutz für das Bundesprogramm Biologische Vielfalt) in ihrem Vortrag „Welche Kommunikation hilft, damit Evaluationsprozesse und -ergebnisse in der Praxis besser genutzt werden?“ dar. Als Fallbeispiel diente dabei die Projektevaluation im Bundesprogramm Biologische Vielfalt. Durch dieses unbefristete Förderprogramm des Bundesumweltministeriums bzw. des Bundesamtes für Naturschutz werden nicht nur klassische Naturschutzmaßnahmen in der Fläche gefördert, sondern auch Umweltbildungs- und Kommunikationsmaßnahmen. Für jedes Projekt erfolgt eine Evaluation seiner zentralen Ziele. Sowohl die Konzeption der Projektevaluation als auch die Auftragsvergabe an Dritte und die Kommunikation der Evaluationsergebnisse sind Aufgaben der Projektplanenden bzw. -durchführenden. Viele Personen empfinden das als Heraus- bis Überforderung, vor allem bei einem sozioökonomischen Evaluationsansatz, der den im Naturschutzbereich tätigen Personen oft inhaltlich fernliegt. Zusätzlich wird eine Konkurrenz um (knappe) Ressourcen empfunden, was teils auf eine geringe Überzeugung vom Nutzen der Evaluation zurückzuführen ist.

Als Orientierung und Hilfestellung haben das Bundesamt für Naturschutz und der DLR-Projektträger im Jahr 2017 einen Leitfaden publiziert, der den Zweck der Projektevaluation verdeutlicht (u.a. Projektzielschärfung, Lernen) und mögliche Herangehensweisen sowie konkrete Praxisbeispiele aus bisherigen Projekten darstellt.

„Kommunikation ist der erste Schritt zur Evaluation“, so die erste Erkenntnis. Der Prozessnutzen der Evaluation muss den Beteiligten bekannt sein. Es muss vermittelt werden, dass Evaluation „etwas Gutes“ ist und die Projektbeteiligten auch etwas angeht.

\section{Weitere Thesen, an denen sich die Diskussion orientierte:}

„Evaluation ist kein Hexenwerk und geht jeden etwas an." Diese Aussage lässt sich sowohl auf die Projektdurchführenden als auch auf die Zielgruppen der Evaluationsergebnisse beziehen. Bei Nicht-Evaluierenden bestehen oft Berührungsängste mit 
dem Thema Evaluation, sie fühlen sich nicht kompetent genug, etwas dazu beizutragen. Nach Überzeugung der Referentin sind aber genau die Personen, die ein Projekt planen und die dessen Kern daher am besten kennen, grob-inhaltlich am besten dazu geeignet, sich zu überlegen, welche Ziele sie verfolgen und anhand welcher Messgrößen sie deren Erreichung messen können. Auf die methodische Expertise von Evaluationsfachleuten kann natürlich nicht verzichtet werden - ihre Beauftragung soll nur nicht als Argument genutzt werden, sich selbst gar nicht mit dem Thema auseinanderzusetzen.

Die Zielgruppen der Evaluationsergebnisse schließlich sollten erkennen, dass Evaluationsergebnisse die Essenz von Projekten darstellen und übertragbare Erkenntnisse ,auf den Punkt bringen“. Dass sie spannend sind und einen Bezug zur eigenen Lebenswelt haben (wenn die Kommunikation gelungen ist). Hierfür ist es nicht erforderlich und manchmal vielleicht sogar kontraproduktiv, wenn der Begriff „Evaluation“ darübersteht. Die These findet in der Runde weitgehenden Zuspruch.

"Je tiefer man im Thema steckt, desto schwerer fällt die Kommunikation auf der Meta-Ebene." Auch zu dieser These herrschte Einigkeit. Der beste Test, ob eine komplizierte Sachlage noch verständlich für die allgemeine Öffentlichkeit ist, sei die Frage, ob man sie einem zehnjährigen Kind erklären könnte, so hatte es die Kommunikationswissenschaftlerin Monika Taddicken bei ihrer Keynote der Jahrestagung auf den Punkt gebracht. Besonders leicht verständlich sind die Ergebnisse, wenn die Kommunikation in der Sprache der Zielgruppen erfolgt. Hilfreich für die „Übersetzung“ kann die Einbindung von Kommunikationsfachleuten sein, die auch bereits in Förderaufrufen, im Budget und in der Zusammenarbeit im Projekt (z.B. Teilnahme an Projektgruppen) berücksichtigt werden sollte. Denn auch falls Kommunikationsfachleute erst zum spätestmöglichen Punkt (dem Projektende) eingebunden werden, um die Evaluationsergebnisse öffentlichkeitswirksam und zielgruppenverständlich zu formulieren (ggf. auch als Video oder ein ähnliches innovatives Format anstelle der sonst üblichen Berichtsform), muss ihre Beauftragung natürlich finanziert werden. Falls dies bei der Projekt- und Budgetplanung nicht berücksichtigt wurde, ist es dazu dann nicht selten zu spät.

Der Nachvollziehbarkeit dienlich sind daneben natürlich auch der Austausch mit Außenstehenden, die Verdeutlichung des Evaluationszwecks, die Formulierung eindeutiger und messbarer Ziele und die Ermittlung eindeutiger Zielgruppen, die durch speziell auf sie zugeschnittene und niedrigschwellige Maßnahmen und Inhalte angesprochen werden sollen.

„Den Prozessnutzen von Evaluation zu kommunizieren stärkt die Akzeptanz, und Akzeptanz ist der Schlüssel zur guten Evaluation." Diese These bezieht sich vor allem auf Projektbeteiligte, deren Bereitschaft zur Mitwirkung bei der Projektevaluation mitunter gering ist. Das ist beispielsweise dann der Fall, wenn die Befürchtung besteht, durch Evaluation kontrolliert zu werden. Ein Hinweis auf die DeGEvalStandards (u.a. die Fairness-Standards) kann hier schon Abhilfe schaffen. Bei formativen Evaluationen wird der Prozessnutzen für Pozessbeteiligte als deutlich höher eingeschätzt als bei summativen Evaluationen, die eher Berichtspflichten und Querschnittsvergleichen dienen und damit u.a. eher den Fördermittelgebenden. 
Darüber, dass „Evaluation durch Kommunikation noch besser in die Gesellschaft getragen werden kann" bestand Einigkeit. Hierzu wurde die Frage aufgeworfen, ob Evaluationen überhaupt für die Gesellschaft oder eher für spezifische Zielgruppen gedacht sind. Könnte ein Kommunikationsleitfaden für verschiedene Ebenen (Laien, Fachleute, Politik) die Außenwirkung verstärken? Wer ist überhaupt zuständig für die Verbreitung von Evaluationsergebnissen? Die einfache Antwort lautet: „Diejenigen, die das Geld haben". Das sind in der Regel die Auftraggebenden bzw. die Fördermittelgebenden. Zur Sichtbarmachung von Best-Practice-Ansätzen wäre eine bei der DeGEval verortete Übersicht bzw. Datenbank hilfreich.

„Die Übertragung großer Ziele auf die eigene Handlungsebene ist eine Herausforderung", so die nächste diskutierte These. Gerade politisch formulierte Strategien (wie z.B. im Umweltbereich die globale Nachhaltigkeitsstrategie oder die Konvention zur biologischen Vielfalt) mit ihren hochaggregierten Indikatoren sind schwer auf die eigene Handlungsebene und den eigenen Evaluationsrahmen zu beziehen. $\mathrm{Ob}$ alle Säulen der Nachhaltigkeit betrachtet werden müssen, sollte durch den Förderaufruf geklärt sein und sich im verfügbaren Budget abbilden. Beim Herunterbrechen von Zielen auf die eigene Handlungsebene hilft es, sich im großen Wirkgefüge zu sehen und überhaupt Wirkzusammenhänge zu erkennen.

Auch die Frage, in welcher Form Evaluationsberichte kommuniziert werden können, wurde diskutiert. Oft benötigen Fördermittelgebende die Berichtsform, in der nachvollziehbar dokumentiert wird, dass ein Zuwendungszweck erfüllt wurde. Hierauf kann daher aus Transparenzgründen nicht verzichtet werden. Darüber hinaus sind aber weitere, interaktive Berichtsformate wie z.B. Videos denkbar, mit denen man gezielt bestimmte Zielgruppen ansprechen kann. Hier seien kreative Ideen gefragt, so die einhellige Meinung. Hilfreich sein könnte ein Wettbewerb für Evaluationsberichte in neuen Formaten und die zentrale Zusammenstellung (z.B. durch die DeGEval) möglicher innovativer Darstellungsformen abseits von langen Textberichten, Tabellen und Diagrammen.

Die gesammelten Anregungen werden genutzt, um die Projektevaluation im Bundesprogramm Biologische Vielfalt und die Arbeit im Arbeitskreis Umwelt der DeGEval weiter voranzubringen.

\section{Ausblick}

Das Thema Naturschutz wird im Arbeitskreis durch die Sprecherfunktion von Christelle Nowack weiterhin als neues Schwerpunktthema ausgebaut. Hierzu zählt, dass 2021 Veranstaltungen zu den Themen Evaluation im Naturschutz und Evaluation von Umweltbildung geplant sind. Hierüber wird über den Newsletter des Arbeitskreises und über die Webseite informiert. 